\title{
BRAIN CANCER
}

\section{Fine-tuning chemoradiotherapy for anaplastic oligodendroglioma}

Anaplastic oligodendroglioma $(\mathrm{AO})$ is a type of brain cancer with a very distinctive histopathology. Apart from harbouring mutations in isocitrate dehydrogenase (IDH) — a feature shared with most types of brain tumours, including lower grade gliomas-AO also presents with frequent losses of the short arm (p) of chromosome 1 and the long $\operatorname{arm}(\mathrm{q})$ of chromosome 19. The standard treatment for $\mathrm{AO}$ is surgical resection followed by radiotherapy; however, up to $70 \%$ of patients have shown durable responses to chemotherapy.

On the basis of this response, nearly 6 years ago, Gregory Cairncross and Martin van den Bent led two independent studies that demonstrated a significant improvement in progressionfree survival (PFS) in patients with $1 \mathrm{p} / 19 \mathrm{q}$ loss who received a combination of radiotherapy and chemotherapy consisting of procarbazine, lomustine, and vincristine (PCV). However, no difference in overall survival was reported. Now, these authors have reported the long-term follow-up of their respective studies in two new papers published in the same issue of the Journal of Clinical Oncology.

In the study led by Cairncross (RTOG 9402), patients with codeleted tumours (loss of $1 \mathrm{p} / 19 \mathrm{q}$ ) were randomly assigned to receive PCV immediately after radiotherapy $(n=59)$ or radiotherapy alone $(n=67)$. After a median duration of follow up of 11.3 years, the median survival was 14.7 years in the PCV plus radiotherapy group versus 7.3 years in the radiotherapy alone group. Median survival was much shorter in patients with noncodeleted tumors, and there was no difference between the two treatment groups (2.6 years and 2.7 years, respectively). In the study led by van den Bent (EORTC 26951), patients with codeleted tumours were randomly assigned to receive PCV within 4 weeks after receiving radiotherapy $(n=43)$ or radiotherapy alone $(n=37)$. Overall survival was not reached in the combination group whereas in the radiotherapy group it was 9.3 years. PFS was also longer after combined therapy (13 years versus 4.1 years). As in the case of the RTOG 9402 study, survival was much shorter in patients with noncodeleted

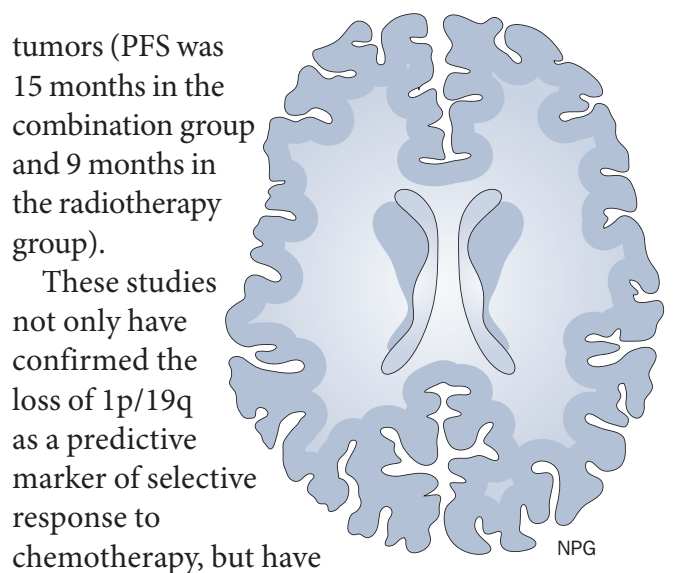

chemotherapy, but have also established a new standard of care for patients with this particular type of AO.

\section{Teresa Villanueva}

Original articles Cairncross, G. et al. Phase III trial of chemoradiotherapy for anaplastic oligodendroglioma: Longterm results of RTOG 9402. J. Clin. Oncol. doi:10.1200/ JC0.2012.43.2674 | van den Bent, M. J. et al. Adjuvant procarbazine, lomustine, and vincristine chemotherapy in newly diagnosed anaplastic oligodendroglioma: Longterm follow-up of European Organisation for Research and Treatment of Cancer Brain Tumor Group study 26951. J. Clin. Oncol. doi:10.1200/JC0.2012.43.2229 